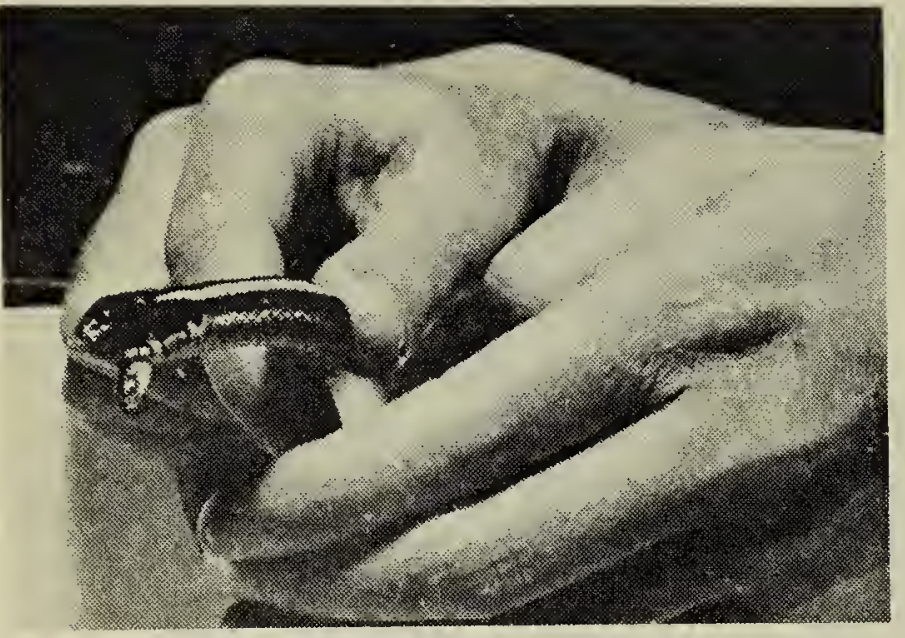

Garter snake eating minnow.

One of the times when the snakes were fed minnows, the fish bowl was placed in the cage at two in the afternoon and the snakes were put into the water. They were observed for a $f \in w$ minutes and then left in the water. At five p.m. they were still as active as at two. In fact, they were both in the bowl completely submerged. Judging from the amount of water in the sand outside the bowl and the amount of sand in the bowl, they had been in and out of the water all afternoon. There were ten of the 40 minnows left (mostly small ones) which would indicate that each snake ate an average of 15 minnows. We offered a few of the remaining minnows to the snakes and they were most cooperative, opening their mouths when they saw our fingers approaching. When we took the fish bowl out of their cage the snakes appeare to be quite excited and darted aroun: in their water dish apparentl searching for more minnows. Th snakes a.te both dead and live min nows. The dead minnows were no taken first although occasionally dead minnow would be eaten whe live minnowis were still available Garter snakes evidently will var their diet to include other than liv food.

During the summer the snake were on a diet of earthworms an frogs, but now are apparently doin well on a diet of raw beef, minnow and other live animals being les readily available. To get the snake to eat raw meat, it was first held an wiggled in front of the snake's nos without it paying any attention $t$ it for a minute or two. Then th snake showed interest by raising it head toward the meat. As the mea was moved gradually back an forth in front of the snake it followe with its head and forepart of it body. After the snake's initerest wa thus aroused the meat was droppe in front of it. The meat fell on th bottom of the cage. With one da? the snake had it in its mouth an proceeded to swallow it. All thi was necessary subsequently to in. terest the snake in pieces of me: was to hold a piece in front of $i$ The snake would grab the meat an eat it.

\title{
How Bright the Stars?
}

\section{by JCHN HODGES, Regina Astronomical Society}

In previous articles, we have described the overall motion of the heavens throughout the year and the grouping of the stars in constellations. We have thus shown how to tell when certain constellations will be visible and how to find any particular star we might be looking for. Another problem of the amateur astronomer is how to tell or compare the brightness of the stars. This is not as difficult as it seems.

Most phenomena in the sky are measured by brightness-the nearness of an approaching planet or a comet, a meteor's behaviour as it streaks across the sky, the outlining of a constellation. The measure of this brightness is called magnitude.

The system now in use to measure a star's brightness had an interestix origin. Hipparchus, the greatest the Greek astronomers, made tu tremendously important contribution to astronomy. He catalogued 1,0 stars, and he divided them in: groups according to their brightnes Six divisions or classes of brillian were established, the first twen bright stars being described as first magnitude and the faintest th were visible to the naked eye sixth magnitude. Note that the sma ler the number, the brighter the sta Ptolemy, who succeeded Hipparchu modified the original catalogue, $b$ little realized that his efforts were remain the standard reference $f_{*}$ some 1,500 years. His catalogue stars was used by Columbus to di 
cover America and by Vasco da Gama to round the Cope of Good Hope.

The original choice by Hipparchus was a very fortunate one as we shall see. When the English astronomer Pogson set about to find how much difference there was between magnitudes in 1850, he was able to report that each magnitude was about two and a half times the previous magnitude. In other words, a magnitude two star is two and a half times fainter than a magnitude one star. It is interesting to note that the ratio between magnitudes one and six is very nearly 100 .

Modern star charts indicate maghitude by the size of the dots used to represent stars. A table of a few stars and their magnitudes follows his article. More precise maps may confuse you because of negative magnitudes. As soon as telescopes were used, it was found that some of the brightest stars were brighter than magnitude one and so a zero magnitude was created. Sirius, the orightest star in the sky, is still orighter so it has a magnitude of -1.6 (the use of a decimal point gives a very precise magnitude). By this scale the full moon has a mag- nitude of -12.6 and the sun a magnitude of -26.8 .

Amateurs observing meteors for the I. G. Y. programme are asked to judge magnitude to half a magnitude. This is not too dificult if you are familiar with the more conspicuous constellations and the stars they contain. Let us take the Little Dipper (Ursa Minor) as an example. Polaris, which forms the first star in the handle, is of 2.0 magnitude. The four stars of the bowl are of second, third, fourth and fifth magnitudes. Do not forget that the brightest of these will have the smallest number. You can tell with the naked eye which is which, and you now have a standard.

You may find a few more constellations easily by starting with the Big Dipper (Ursa Major) and using the following scheme. The pointer stars are five degrees apart, and six times this distance away or thirty degrees lies Polaris. The bottom of the bowl in a direction away from the handle points to Castor in the constellation Gemini. The two stars on the handle side of the bowl point to Regulus in Leo. Following the curve of the handle will guide you to Arcturus in Bootes.
TABLE OF MAGNITUDES

Magnitude Constellation

$\begin{array}{ll}2.0 & \text { Little Dipper (Ursa Minor) } \\ 1.9 & \text { Big Dipper (Ursa Major) } \\ 1.0 & \text { Twins (Gemini) } \\ 1.0 & \text { Lion (Leo) } \\ 0 & \text { Shepherd (Bootes) }\end{array}$

2.0 Little Dipper (Ursa Minor)

1.0

0

Shepherd (Bootes)

Month when overhead at 9:00 p.m.

June, December

April

February

April

June

\section{A Record of Boloria toddi toddi double brooded at the Pas, Manitoba}

by WALTER KRIVDA, the Pas, Manitoba

I have collected butterflies in the area of the Pas, Manitoba for almost ten years now, but this is the first year that I have found Boloria toddi toddi double broooded.

The typical brood appears about the middle of June. The flight period begins with a preponderance of males, followed by the gradual appearance of the females. As the flight ends, fresh females can still be taken on the wing, but males are tattered and worn. The flight is usually over by the first week of July. The females are the last to
The best spot for this small Fritillary in the Pas area is Devon Park, which is almost native "lawn" with such plant species as Anemone riparia, Sisyrinchium angustifolium, Astragalus goniatus, Antemmaria sp. (sterile rosettes), Vicia cracca and Poa palustris. This "lawn" is mowed infrequently, permitting the establishment and persistence of a fine colony of Boloria toddi. They probably feed on Viola rugulosa growing among the rocks along the Saskatchewan River.

On August 2, 1957, near the east gate of the golf course, in a low, 